\title{
Hydroxysteroid dehydrogenase HSD1L is localised to the pituitary- gonadal axis of primates
}

\author{
A Daniel Bird ${ }^{1, *,+}$, Spencer Greatorex ${ }^{1,+}{ }^{\text {, David Reser }}{ }^{2}$, Gareth G Lavery ${ }^{3,4}$ and \\ Timothy J Cole ${ }^{1}$
}

${ }^{1}$ Department of Biochemistry and Molecular Biology, Monash University, Melbourne, Victoria, Australia 2Department of Physiology, Monash University, Melbourne, Victoria, Australia

${ }^{3}$ Institute of Metabolism and Systems Research, 2nd Floor IBR Tower, University of Birmingham, Birmingham, UK ${ }^{4}$ Centre for Endocrinology, Diabetes and Metabolism, Birmingham Health Partners, Birmingham, UK

*(A D Bird is now at the Centre for Endocrinology and Metabolism, Hudson Institute of Medical Research,

Monash Medical Centre, Clayton, Victoria, Australia)

${ }^{\dagger}$ (A D Bird and S Greatorex contributed equally to this work)

\author{
Correspondence \\ should be addressed \\ to $\mathrm{T} J$ Cole \\ Email \\ tim.cole@monash.edu
}

\begin{abstract}
Steroid hormones play clinically important and specific regulatory roles in the development, growth, metabolism, reproduction and brain function in human. The type 1 and 2 11-beta hydroxysteroid dehydrogenase enzymes (11 $\beta$-HSD1 and 2) have key roles in the pre-receptor modification of glucocorticoids allowing aldosterone regulation of blood pressure, control of systemic fluid and electrolyte homeostasis and modulation of integrated metabolism and brain function. Although the activity and function of $11 \beta$-HSDs is thought to be understood, there exists an open reading frame for a distinct $11 \beta$ HSD-like gene; HSD11B1L, which is present in human, non-human primate, sheep, pig and many other higher organisms, whereas an orthologue is absent in the genomes of mouse, rat and rabbit. We have now characterised this novel HSD11B1L gene as encoded by 9 exons and analysis of EST library transcripts indicated the use of two alternate ATG start sites in exons 2 and 3, and alternate splicing in exon 9. Relatively strong HSD11B1L gene expression was detected in human, non-human primate and sheep tissue samples from the brain, ovary and testis. Analysis in non-human primates and sheep by immunohistochemistry localised HSD11B1L protein to the cytoplasm of ovarian granulosa cells, testis Leydig cells, and gonadatroph cells in the anterior pituitary. Intracellular localisation analysis in transfected human HEK293 cells showed HSD1L protein within the endoplasmic reticulum and sequence analysis suggests that similar to $11 \beta \mathrm{HSD} 1$ it is membrane bound. The endogenous substrate of this third HSD enzyme remains elusive with localisation and expression data suggesting a reproductive hormone as a likely substrate.
\end{abstract}

\section{Key Words}

- hydroxysteroid dehydrogenases

- pituitary

- gonads

- ovary

- testis

\section{Introduction}

The short-chain alcohol dehydrogenase/reductase

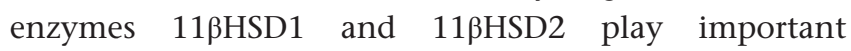
intracellular roles in mammals, regulating tissue availability of physiologically relevant glucocorticoid $(1,2)$. They are members of the large short-chain dehydrogenase/reductase (SDR) family of enzymes that are $\mathrm{NAD}^{+}$or $\mathrm{NADP}^{+}$dependent oxidoreductases, typically 250-350 amino acids in length (3). SDR enzymes have a wide range of substrates including steroids, aromatic compounds and xenobiotics. The human genome

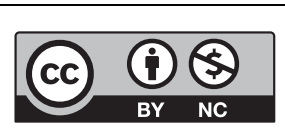


encodes up to 63 different SDR enzymes with many uncharacterised for substrate and function in vivo (4). All SDR family members share a residue homology of only 20-40\%. They have two conserved motifs, a 'GXXXGXG' nucleotide cofactor $\left(\mathrm{NAD}^{+} / \mathrm{NADP}^{+}\right)$binding domain and an 'YXXXK' catalytic active site domain (4).

$11 \beta \mathrm{HSD} 1$ and $11 \beta \mathrm{HSD} 2$ carry out largely opposing roles in the preceptor modification of corticosteroids. 11ßHSD1, encoded by the HSD11B1 gene and located on chromosome 1q32-41 in humans, is a bidirectional enzyme but normally acts as an oxidoreductase in vivo to drive formation of the active glucocorticoid cortisol. Located in the endoplasmic reticulum (ER) membrane, it requires NADPH provided by the enzyme hexose-6phosphate dehydrogenase (H6PDH) for activity. 11 $\beta$ HSD1 is expressed widely in metabolic tissues such as liver, muscle, adipose and kidney, and also in many specific regions of the brain (5). In contrast, $11 \beta \mathrm{HSD} 2$, encoded by the HSD11B2 gene and located on chromosome 16q22.1 in humans, is a unidirectional dehydrogenase producing the inactive 11-keto metabolite cortisone from cortisol (5). 11ßHSD2 has a more restricted expression pattern, and is detected predominantly in aldosterone target tissues where it prevents inappropriate activation of the mineralocorticoid receptor (MR) by glucocorticoids by efficiently converting cortisol to inactive cortisone $(6,7)$.

Inappropriate 11ßHSD1 expression and activity is implicated in human disease such as obesity, insulin resistance and metabolic syndrome. Several 11ßHSD1 selective inhibitors have been developed by the pharmaceutical industry and are currently being tested in Phase II trials to treat type- 2 diabetes and obesity $(8,9)$.

A search of the annotated human genome for other potential SDR family members detected an 11ßHSD1like gene termed HSD11B1L on human chromosome 19p13.3. HSD11B1L is composed of 9 exons, stretching over $7.5 \mathrm{~kb}$ and analysis of EST library transcripts indicates the use of two alternate ATG start sites in exons 2 and 3, and alternative RNA splicing in exon 9. HSD11B1L has also been referred to as SCDR10B, and an SDR enzyme family member, and showed that it was highly expressed in the brain, with evidence that the when expressed in vitro the enzyme possesses very weak dehydrogenase activity in inactivate glucocorticoid (10). Surprisingly phylogenetic analysis of this gene in other mammalian genomes revealed that it is completely absent from all rodent genomes and also the rabbit genome (11). From an increasingly more detailed annotation of mammalian genomes the number of primate-specific or primate-restricted genes is growing
$(12,13,14)$. The lack of paralogues in the common laboratory rodent models makes characterisation of these new genes much more difficult and loss-offunctional analysis almost impossible. We have therefore extended our understanding of HSD11B1L expression, and tissue and cell localisation by analysis in the nonhuman primate marmoset and in the sheep. We reveal strong levels of expression in the pituitary gland and the ovary, with moderate-to-low expression in other organs, including the testis. Immunohistochemistry using a polyclonal antibody to human HSD1L localised protein to gonadotroph cells in the anterior pituitary and the granulosa cells of the ovary.

\section{Materials and methods}

\section{Animals, tissue samples and cell lines}

Collection of non-human primate, sheep and mouse tissues were approved in advance by the Monash University Animal Ethics Committee, and conformed to the Australian National Health and Medical Research Council Guide for Care and Use of Laboratory Animals, which strongly encourages use of archived and scavenged tissues. Tissue samples were collected from adult and foetal sheep, the non-human primate marmoset and macaque, and from mice, then they were snap frozen for RNA isolation or fixed in 4\% paraformaldehyde (PFA) overnight at $4^{\circ} \mathrm{C}$ for histology and analysis by immunohistochemistry. Human HEK293 cells were maintained in culture in DMEM/high glucose medium with $2 \mathrm{mM}$ glutamine, $10 \%$ FCS.

\section{Bioinformatics and protein structure modelling}

Sequence alignment of human HSD11B1L and 11ßHSD1 were performed using the Clustral Omega (EMBL-EBI, http://www.ebi.ac.uk/Tool/msa/clustalo) software package. Amino acid sequences were obtained from the NCBI database for human HSD11B1L (NM_198706) and 11ßHSD1 (NP_005516.1). Signal peptide prediction for human HSD11B1L was determined with the SignalIP4.1 software package (http://www.cbs.dtu.dk/services/ SignalIP/). Three-dimensional modelling was performed using the Phyre2 software package to predict the 3D structure of human HSD11B1L (286 amino acid form) and was compared to the solved 3D structure of human $11 \beta \mathrm{HSD} 1$ that was obtained from the protein database (http://www.rcsb.org/pdb/), 11ßHSD1 identifier 1XU9.

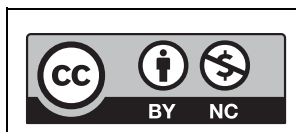

This work is licensed under a Creative Commons Attribution-NonCommercial 4.0 International License. 


\section{RNA extraction, CDNA synthesis and analysis by qPCR} and droplet-digital PCR

Total RNA was isolated from tissue samples using TRIzol reagent (Invitrogen) as per the manufacturer's instructions. cDNA was then synthesised from total RNA using the QuantiTect reverse transcriptase kit (Qiagen) as per the manufacturer's instructions. Expression levels of mRNA in adult sheep and human tissue or cell samples were analysed using a Rotor-Gene 3000 PCR (Qiagen), with each biological replicate assayed in triplicate using SYBR Green qPCR SuperMix (Invitrogen). Rps29 and 18S-rRNA levels were used as a normalising RNA control. Dissociation curves were performed for each $\mathrm{QPCR}$ experiment to ensure that a single PCR product had been amplified per primer set and PCR products were also sequenced to verify fragment identity. Differential expression was determined using the comparative delta-delta CT method (15). Foetal sheep gene expression levels were analysed using the QX-200 droplet-digital PCR system (Biorad). For all PCR-based analyses, primers corresponding to mRNAs of interest were designed to overlap exon-exon boundaries and therefore prevent amplification of genomic DNA.

\section{Immunohistochemistry and immunofluorescence}

Marmoset, macaque and sheep tissue samples were immersion-fixed in $4 \%$ paraformaldehyde overnight at $4^{\circ} \mathrm{C}$, then processed and embedded into paraffin. Paraffin sections with a thickness of $5 \mu \mathrm{m}$ were cut and mounted on slides, blocked with an appropriate animal serum (5\%), then immunostained with donkey anti-HSD1L (sc-1004, Santa Cruz) primary antibodies overnight at $4^{\circ} \mathrm{C}$. Supplementary Table 1 (see section on supplementary data given at the end of this article) provides details of all antibodies used in this study. Primary antibodies were then detected by subsequent application of biotinylated secondary antibodies, Streptavidin-HRP (Invitrogen), and DAB (3,3'-diaminobenzidine) solution (Dakocytomation, Glostrup, Denmark). All immunohistochemistry images were obtained using the Olympus brightfield microscopy. For immunofluorescence, $5 \mu \mathrm{m}$ thick paraffin sections were cut and mounted on slides blocked with an appropriate animal serum (5\%), then immunostained with primary antibodies (donkey anti-HSD1L; rabbit anti-FoxL2, LH and Cyclin D2 antibodies) overnight at $4^{\circ} \mathrm{C}$. Sections were washed and stained with fluorescent secondary antibodies (goat anti-donkey 488 and donkey anti-rabbit 555, Life Technologies). Sections were counterstained with Hoechst 33342 (Sigma), then mounted in fluorescent mounting medium (Dako). All fluorescent images were taken using an Olympus fluorescent microscope (IX71) and images were prepared using ImageJ software.

\section{Intracellular localisation}

HEK293 cells were grown at $37^{\circ} \mathrm{C}$ and $5 \% \mathrm{CO}_{2}$ in DMEM High Glucose: F12 supplemented with 10\% FBS, $2 \mathrm{mM}$ glutamine and $1 \%$ penicillin-streptomycin. Once confluent, cells were detached using trypsin and resuspended in pre-warmed media to a cell density of $2 \times 10^{5} \mathrm{cells} / \mathrm{mL}$, then plated out in a 6 -well dish with $1 \mathrm{~mL}$ per well. Cells were left overnight to re-attach and reach approximately $80-90 \%$ confluency, then washed and treated with $1 \mu \mathrm{g}$ of either pc-DNA6B-HSD11B1L, pcDNA6B-HSD11B1L/mCherry or control pcDNA6B (No insert) DNA in $8 \mu \mathrm{L}$ of lipofectamine 2000 diluted in $500 \mu \mathrm{L}$ of serum-free media, then incubated overnight. Cells were then fixed in 2\% PFA, permeabilised using 1\% SDS and then immunostained with primary antibodies (rabbit anti-GRP78 and a mouse anti-HSD1L monoclonal antibody produced in the laboratory to a hHSD1L peptide), then washed and detected with secondary antibodies (anti-rabbit IgG alexa-488 and anti-mouse IgG2a alexa-555 (Life Technologies)). Cells were then counterstained with Heochst 33342 and mounted using DAKO fluorescent mounting media. All cells were imaged using the Olympus IX71 fluorescent microscope.

\section{Statistical analysis}

GraphPad Prism software was used to analyse the results of all experiments. The statistical significance from qPCR analyses was determined using a two-tailed unpaired Student's $t$-test. For all analyses, the statistical significance was set at $P<0.05$, with all error bars depicting standard error of the mean (S.E.M.).

\section{Results}

\section{Analysis of the putative human SDR enzyme encoded by the HSD11B1L gene}

A bioinformatics search of the annotated human genome for members of the SDR enzyme superfamily related to $11 \beta H S D 1$ identified an 11ßHSD1-like annotated gene on human chromosome 19p13.3 termed HSD11B1L. This gene was composed of 9 exons, covering $7.5 \mathrm{~kb}$ of genomic

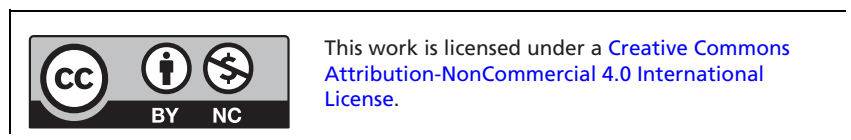


DNA and analysis of EST library transcripts indicated the use of two alternate ATG translation start sites in exons 2 and 3, respectively. Furthermore, alternate RNA splicing in exon 9 generated two isoforms, an A and B form that encode variable C-terminal ends (Fig. 1A). One publication exists on the analysis of this gene, referred to as SCDR10B, an SDR enzyme family member, which suggested the human gene was highly expressed in the brain and weakly expressed in other organs (10). Analysis was, however, very limited, and also suggested that the enzyme may have a very weak substrate dehydrogenase activity for the steroid cortisol in vitro. Phylogenetic analysis of this gene in other genomes revealed that it is completely absent from all rodent genomes and is also absent in the rabbit genome (11). It has an orthologue in all primate, cow, ovine and dog genomes, and two similar genes exist in the zebrafish genome. The syntenic region of human chromosome 19p13.3 in the mouse genome

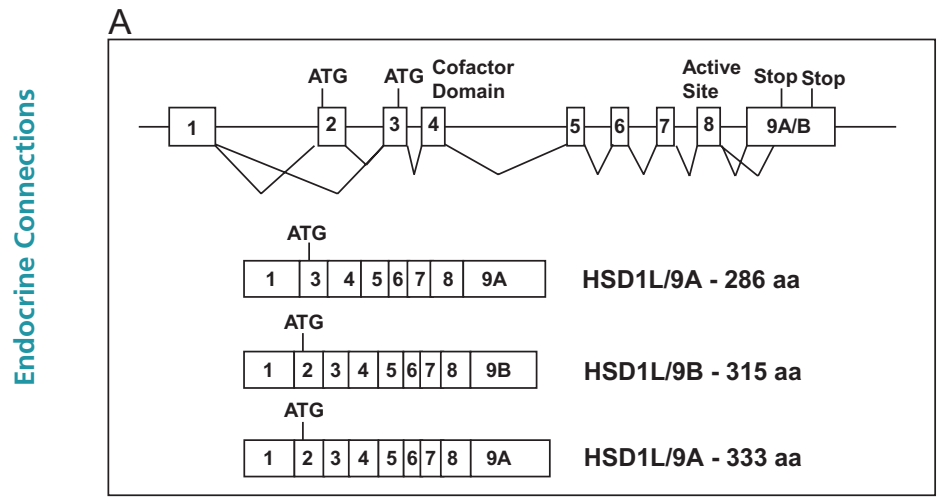

B

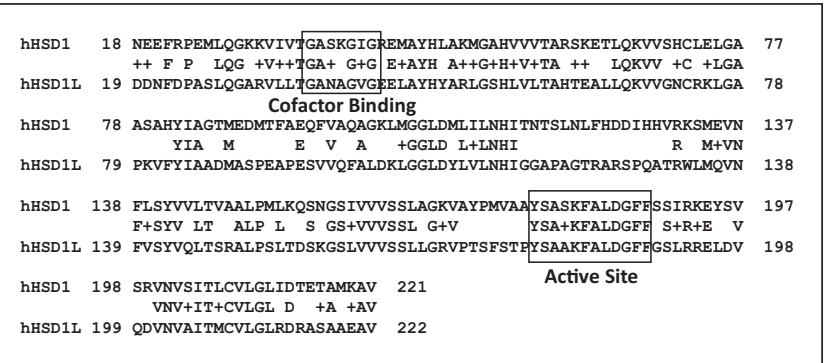

\section{Figure 1}

The exon/intron structure of the human HSD11B1L gene and the amino acid sequence comparison of human $11 \beta \mathrm{HSD} 1$ and HSD1L. (A) The exon/ intron structure of the human HSD11B1L gene on chromosome 19p33.3. Positions of two alternate ATG start sites, the cofactor binding domain and active site are indicated above exons. Alternate splicing of exons is able to generate three major protein isoforms of 286, 315 and 333 amino acids. (B) Amino acid sequence comparison of human 11 $\beta \mathrm{HSD} 1$ and HSD1L showing the known positions of the NAD/NADP binding and active sites for $11 \beta \mathrm{HSD} 1$ and HSD1L (boxed). Conserved and similar (+) residues across the two sequences are also indicated. (located on chromosome 17) has the same surrounding cluster of genes (SAFB2, SAFB, RPL36, LONP1) but remarkably is completely missing an annotated $H S D 11 B 1 L$ gene between SAFB and RPL36. Comparison of the amino acid sequence of the encoded protein for the HSD11B1L gene to human $11 \beta \mathrm{HSD} 1$ and $11 \beta \mathrm{HSD} 2$ showed a striking level of homology and the strong conservation of the $\mathrm{NAD}^{+} / \mathrm{NADP}^{+}$nucleotide binding and dehydrogenase/ reductase catalytic site domains. HSD11B1L is most similar in sequence to human 11ßHSD1 (Fig. 1B) with an approximate $40 \%$ amino acid identity and a strong conservation of the key catalytic enzyme domain and cofactor binding site (Fig. 1B). These analyses imply that $H S D 11 B 1 L$ represents a species-restricted member of the SDR superfamily that is absent in all rodent genomes, and in humans, it may play a critical role in modulating availability of an as yet unidentified substrate in vivo. The sequence of the 286 amino acid HSD1L9A isoform was used with the 'Pymol' molecular modelling programme (PyMOL Molecular Graphics System, Version 1.8 Schrödinger, LLC) to compare a predicted 3D structure of human HSD1L to the known structures of human

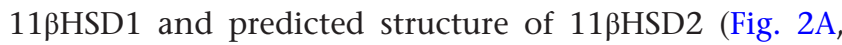
B and C). Predicted $\alpha$-helices and the $\beta$-sheet 'Rossmann' fold, a common feature of all SDR enzymes (4), were very similar to the determined structure of murine $11 \beta \mathrm{HSD} 1$ (16) and somewhat similar to that for predicted 11ßHSD2 structure (Fig. 2A, B and C, shown in magenta and red). Positioning of residues important at the catalytic site was also very similar between 11ßHSD1 and HSD1L9A (Fig. 2, panels B and C, shown in dark blue). HSD1L also contained a putative ER localisation signal sequence at the N-terminus (Fig. 2C in light blue). Charged amino acids at Tyr-178 and Lys-182 were found in the predicted catalytic domain (Fig. 2D, arrow 1), orientated in such a way that their side chains pointed towards the predicted cofactor binding motif (Fig. 2D, arrow 2). Analysis of HSD1L hydrophobicity using a surface map (Fig. 2E red residues) showed that the charged amino acids pointed towards a hydrophobic pocket (Fig. 2E arrow).

\section{HSD11B1L mRNA is expressed in the pituitary, ovary, lung and gastrointestinal tract}

Previous studies indicated that human HSD11B1L mRNAs were strongly expressed in the brain and weakly in other organs (10). We assessed human HSD11B1L expression in total RNA from various human tissues and cells by RT-PCR (Fig. 3A). Using PCR primers from exons 6 and 8 

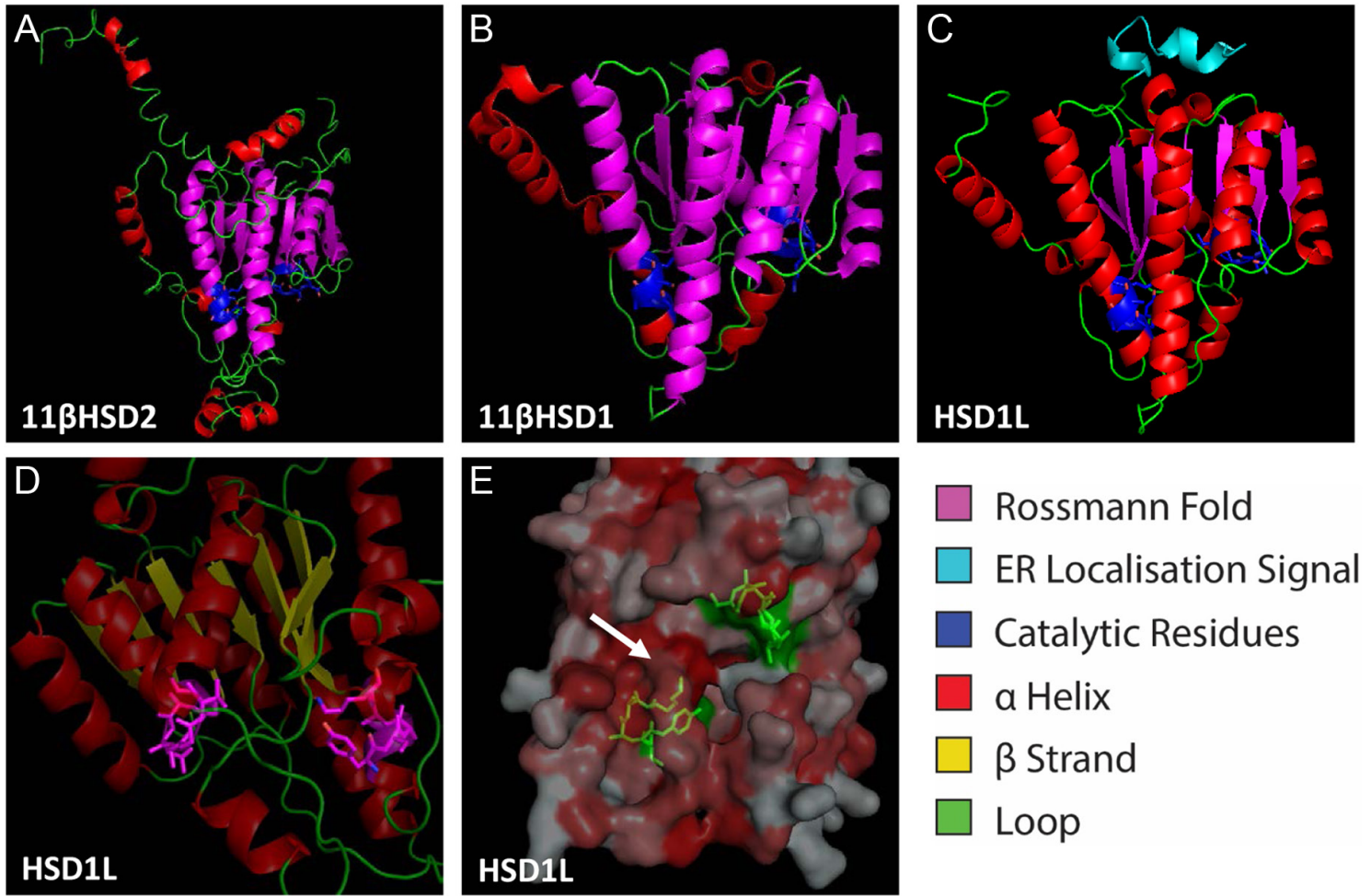

\begin{abstract}
Figure 2
Structural modeling of the open reading frame of the human HSD11B1L gene with its closest homologues $11 \beta \mathrm{HSD} 1$ and $11 \beta \mathrm{HSD} 2$. The three-dimensional

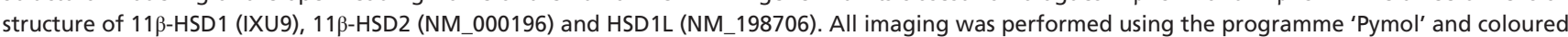
as described unless stated otherwise. Predicted structures of 11 $\beta \mathrm{HSD} 2$ and 11ßHSD1L were produced using the Phyre2 software suite. (A) The predicted $3 \mathrm{D}$ structure of $11 \beta \mathrm{HSD} 2$ produced using Phyre2 at $>90 \%$ confidence in $74 \%$ of residues. (B) Depicts the solved structure of $11 \beta \mathrm{HSD} 1$. (C) The predicted 3D structure of HSD1L produced using Phyre2 at $>90 \%$ confidence in $74 \%$ of residues. (D) Shows the predicted substrate binding site of $11 \beta \mathrm{HSD} 1 \mathrm{~L}$ with both catalytic residues represented as stick diagrams. (E) Shows a hydrophobicity surface structure of the predicted HSD1L structure. Red regions indicate hydrophobic areas whereas white indicated hydrophilic regions. Both the catalytic and cofactor binding domains are shown in green and the respective side chains are represented as stick diagrams.
\end{abstract}

of the $h H S D 1 L$ gene we detected moderate expression in total RNA from whole brain in agreement with previous reports (10). We detected stronger bands for HSD11B1L transcripts in the ovary, testis and lung, and weaker expression in total RNA from human kidney (Fig. 3A). We then compared mRNA levels of hHSD1L to h11 $\beta$ HSD1 and h1 $1 \beta$ HSD 2 in total RNA from human colon. $11 \beta$ HSD2 mRNA levels were as expected very high, with hHSD1L and $11 \beta$ HSD1 almost undetectable (Fig. 3B). A similar analysis in total RNA from human brain and ovary showed similar levels of expression in the ovary, and similar mRNA levels for $11 \beta$ HSD1 and hHSD1L in the brain (Fig. 3C). To allow a broader assessment of the expression of HSD1L in the mammalian body, we measured mRNA levels in total RNA from various organs of the sheep. Total RNA was prepared from the major organs of the foetal sheep (female, day 150 , just prior to birth) and mRNA levels compared for

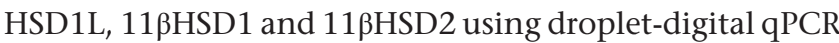
(Fig. 4A). Highest levels of HSD1L mRNA were detected in ovary, pituitary, colon and lung, with lower levels detected in other organs analysed. In the adult female sheep, much higher levels of HSD1L mRNA were detected in pituitary and ovary compared to the lung (Fig. 4C).

\section{Localisation of HSD1L protein in the ovary}

The cellular localisation of HSD1L was assessed in the ovary and testis of the non-human primate marmoset by immunohistochemistry using a commercial anti-human HSD1L polyclonal antibody. Strong immunostaining for HSD1L was detected in developing follicles of the ovary at all stages of ovarian follicle development (Fig. 5A and D). Also shown are negative controls with no primary antibody (panel B) and an immunizing peptide, preincubation control (panel C). The mouse ovary, that should not express a HSD1L protein, was used as an additional negative control for immunostaining and showed no specific staining in the mouse ovary above

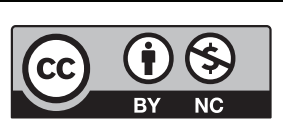

This work is licensed under a Creative Commons Attribution-NonCommercial 4.0 International License. 


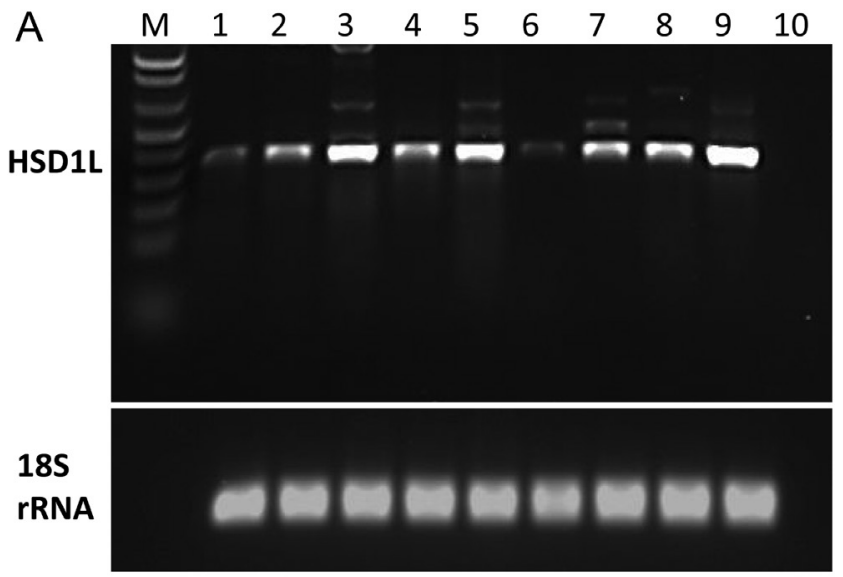

B

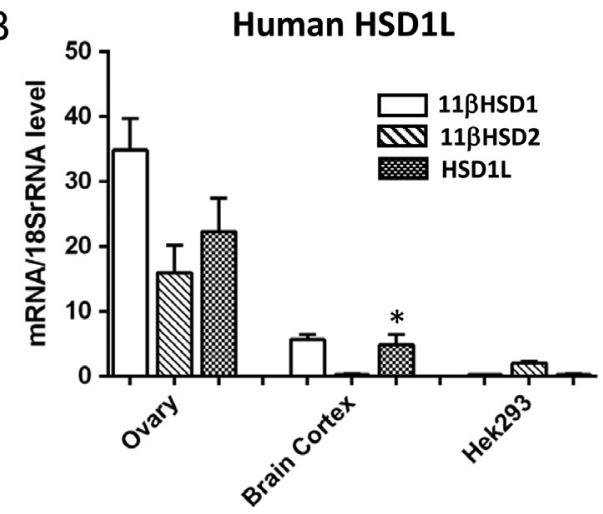

Figure 3

Detection of HSD11B1L mRNA in total RNA from human tissues and cell lines. (A) Detection of HSD11B1L mRNA by RT-PCR in CDNA from human kidney (lane 1), lung (lane 2), ovary (lanes 3 and 5), testis (lanes 4 and 6) and whole brain (lanes 7-9). (B) Relative mRNA levels of 11 $\beta$ HSD1, $11 \beta \mathrm{HSD} 2$ and HSD1L determined by qPCR in total RNA from human ovary, brain and the HEK293 cell line $(n=3)$. RNA levels of HSD1L compared to $11 \beta \mathrm{HSD} 2$ in brain cortex, $* P<0.05$. background (Fig. 5E and F). Staining within the marmoset ovary was specific for the granulosa cell layer of the follicle and interestingly also for the oocyte within the follicle (arrow, Fig. 5D). An identical staining pattern was also observed in the ovary from the adult sheep (data not shown). Immunohistochemistry with sections of the marmoset testis was also analysed and detected staining in cells resembling Leydig cells (Fig. 5, panel G and negative control, panel $\mathrm{H}$ ). To positively identify the cell type specifically labelled in the marmoset ovary, we performed double-immunofluorescence for HSD1L with two known granulosa cell markers, cyclin D2 and FoxL2 (Fig. 6). Immunostaining for HSD1L was co-localised in granulosa cells with both cyclin D2 (Fig. 6A, B and C) and FoxL2 (Fig. 6D, E and F), clearly identifying HSD1L expression specifically to the cytoplasm of ovarian granulosa cells.

\section{Localisation of HSD1L protein within the anterior pituitary}

The cellular localisation of HSD1L in the pituitary was also assessed by immunohistochemistry in the marmoset. Immunostaining was detected in a small subset of cells and only in anterior pituitary (Fig. 7). No staining was detected in regions of the posterior pituitary (data not shown). In the anterior pituitary, strong cytoplasmic staining was observed in scattered cells throughout the anterior lobe (Fig. 7A and D). To identify the particular cell type stained for HSD1L double-immunofluorescence was performed with antibody markers specific for pituitary somatotrophs (growth hormone), lactotrophs (prolactin), gonadotrophs (luteinizing hormone (LH)) and corticotrophs (melanocyte-stimulating hormone) (Fig. 7E,

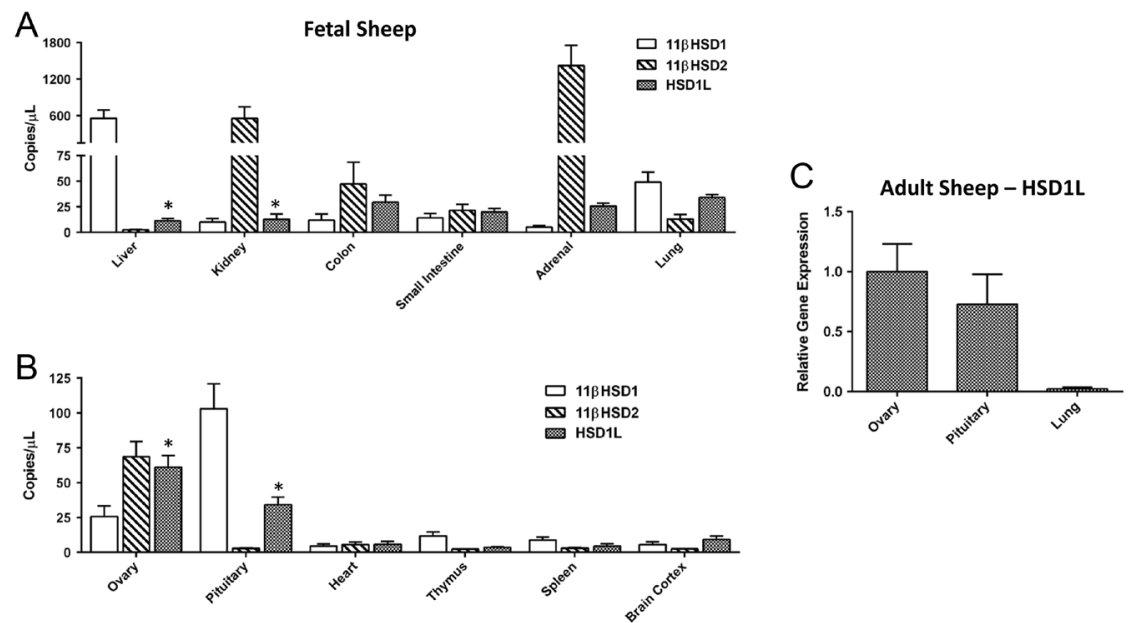

Figure 4

Levels of Sheep $11 \beta \mathrm{HSD} 1,11 \beta \mathrm{HSD} 2$ and HSD1L mRNA in total RNA isolated from female foetal (156 day old) and female adult sheep tissues determined by drop-digital PCR $(n=3)$. Values shown are absolute relative transcripts/10 ng total RNA for (A) foetal liver, kidney, colon, small intestine, adrenal and lung. (B) Foetal ovary, pituitary, heart, thymus, spleen and brain cortex. (C) Adult ovary, pituitary and lung. Error bars are S.E.M., $n=3$. RNA levels of HSD1L compared to $11 \beta$ HSD 1 in liver, $* P<0.01$, RNA levels of HSD1L compared to $11 \beta \mathrm{HSD} 2$ in kidney and adrenal, $* P<0.01$, RNA levels of HSD1L compared to $11 \beta$ HSD 1 in ovary, ${ }^{*} P<0.05$, and RNA levels of HSD1L compared to $11 \beta \mathrm{HSD} 1$ and $11 \beta \mathrm{HSD} 2$ in pituitary, ${ }^{*} P<0.01$. 

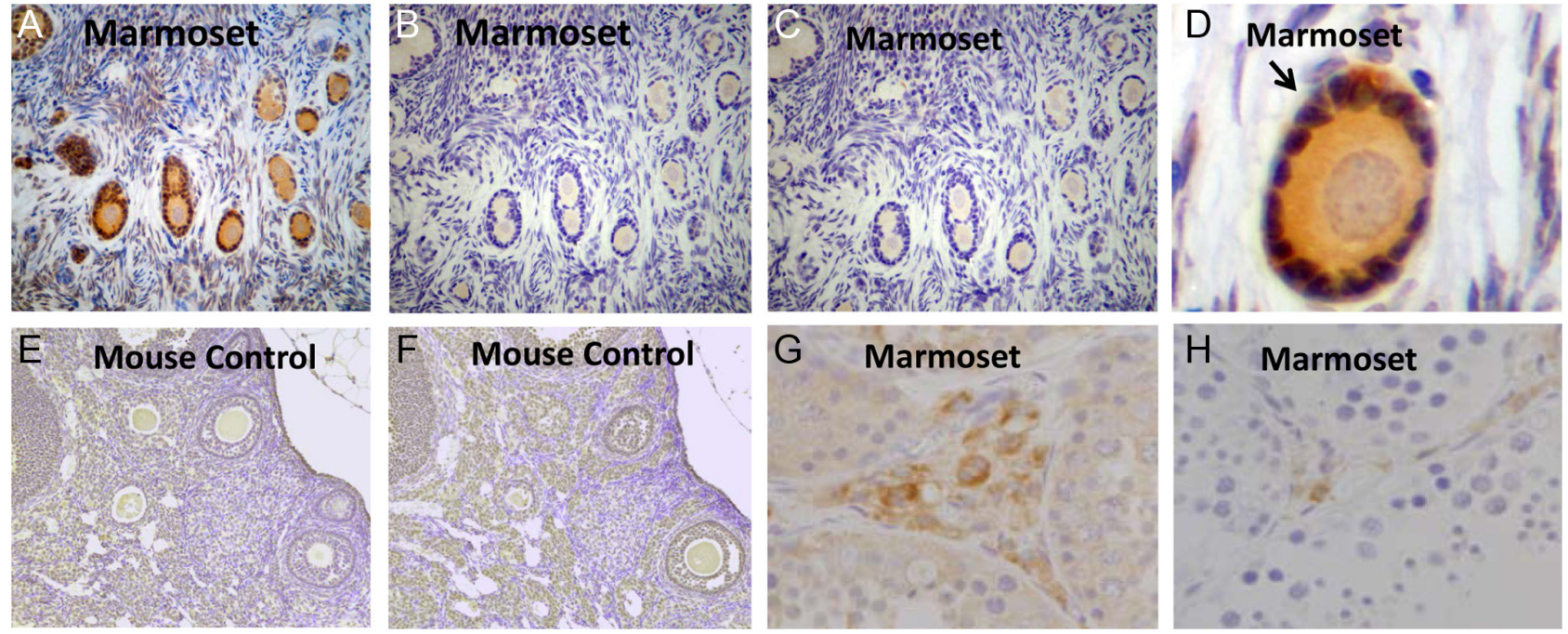

Figure 5

Localisation of HSD1L by immunohistochemistry in the ovary and testis from the non-human primate adult marmoset. (A) HSD1L in marmoset ovary (N16 HSD1L peptide polyclonal antibody) $\times 10$. (B) Marmoset ovary no primary antibody control. (C) Marmoset ovary immunising peptide pre-incubationnegative control. (D) HSD1L in Marmoset ovary (N16 HSD1L peptide polyclonal antibody) ×40. (E) Mouse ovary (N16 HSD1L peptide polyclonal antibody) $\times 10$. (F) C Marmoset ovary no primary antibody negative control. (G) HSD1L in Marmoset testis (N16 HSD1L peptide polyclonal antibody) $\times 10$.

(H) Marmoset testis no primary antibody control.

F, G and H). There was no co-localisation of HSD1L (green colour) with markers for somatotrophs, corticotrophs or lactotrophs (red colour), but remarkably there was close to
100\% co-localization of HSD1L with LH (panel H, merged yellow colour), marking these HSD1L-specific cells as pituitary gonadotrophs.
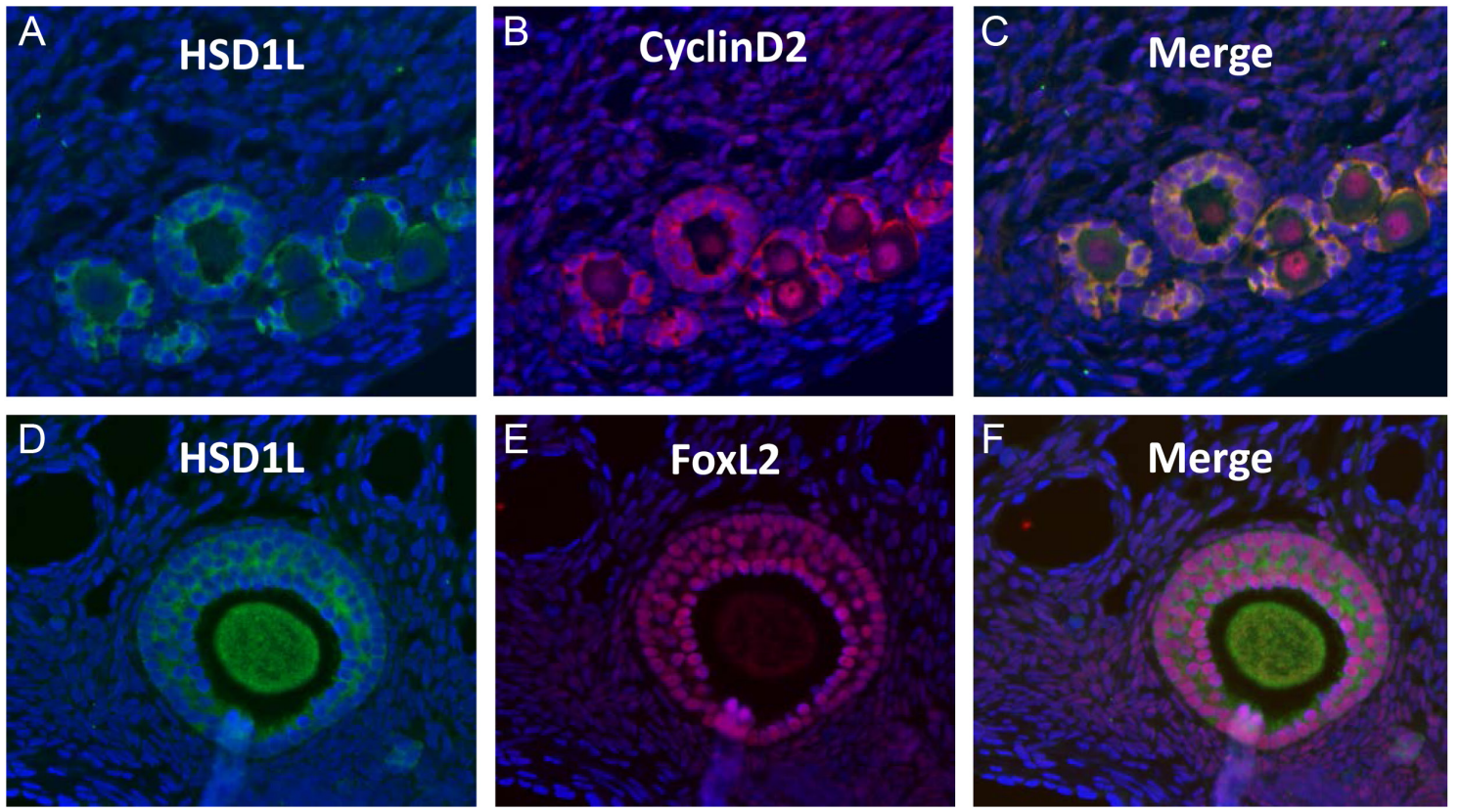

Figure 6

Localisation of HSD11B1L protein to granulosa cells of the adult marmoset ovary by double-immunofluorescence. (A, B and C) DoubleImmunofluorescence for HSD1L (green) and cyclinD2 (red) in the adult marmoset ovary. CyclinD2 was used a marker of follicle granulosa cells and shows co-localisation with HSD1L to the cytoplasm of granulosa cells (C; yellow, Merge). (D, E and F) Double-immunofluorescence for HSD1L (green) and FoxL2 (red) in the adult marmoset ovary. FoxL2 was used a nuclear marker of follicle granulosa cells. HSD1L and FoxL2 were strongly co-localised to the cytoplasm and nucleus, respectively of follicle granulosa cells (F; Merge). Scale bars: A, B, C, D, E and F: $80 \mu \mathrm{m}$.

\begin{tabular}{|lr}
\hline http://www.endocrineconnections.org & ○ 2017 The authors \\
DOI: 10.1530/EC-17-0119 & Published by Bioscientifica Ltd
\end{tabular}

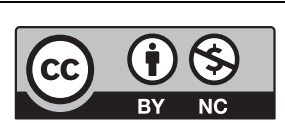

This work is licensed under a Creative Commons Attribution-NonCommercial 4.0 International License. 

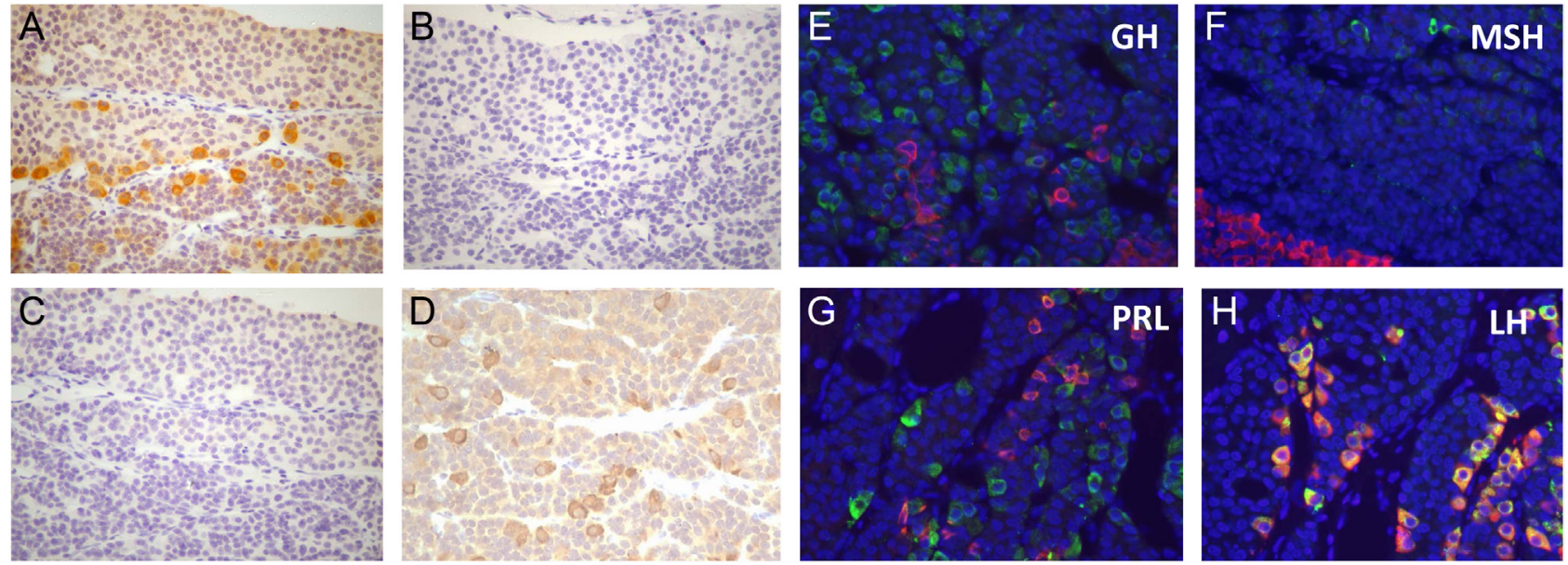

\section{Figure 7}

Localisation of HSD11B1L by immunohistochemistry and double-immunofluorescence in the anterior pituitary of non-human primate adult marmoset. (A, B, C and D) Immunohistochemistry for HSD1L (DAB, brown stain) in the adult marmoset anterior pituitary. (A) HSD1L N16 primary antibody (SC-244840) 20x. (B) no primary antibody (N16-SC) control. (C) Immunising peptide (SC-244840) pre-incubation (before primary antibody) control. (D) 11ßHSD1L N16 primary antibody 40x. (E, F, G and H) Double-immunofluorescence for HSD1L (green fluorescence) with either growth hormone (panel E, GH - red), $\alpha M S H$ (panel F, MSH - red), prolactin (panel G, PRL - red) or Luteinizing hormone (panel H, LH - red). Co-localisation is shown as yellow fluorescence.

\section{HSD1L protein is localised to the cytoplasmic endoplasmic reticulum}

The level of conservation between HSD11B1 and HSD11B1L on a protein level is exceptionally high particularly in amino acid sequences that form the secondary structure involved in the Rossmann fold. Further levels of conservation are observed at the N-terminal end of HSD11B1L showing a string of hydrophobic amino acids that suggested the possibility of a localisation signal and/or transmembrane domain. Analysis of the HSD1L amino acid sequence using
A
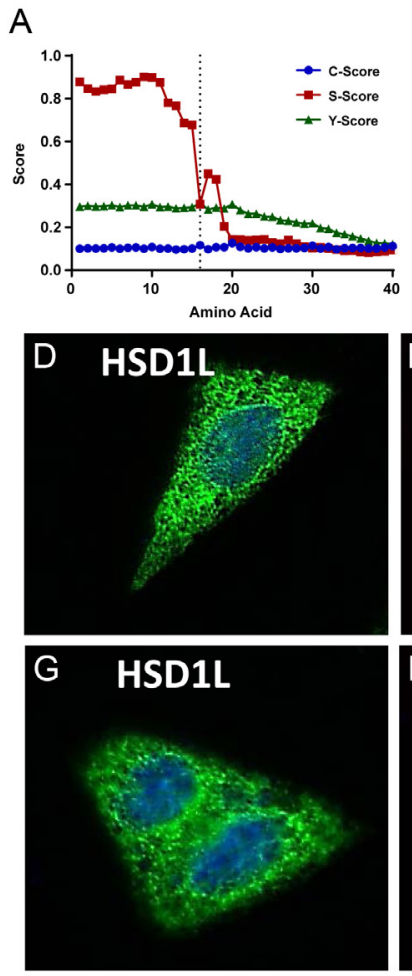
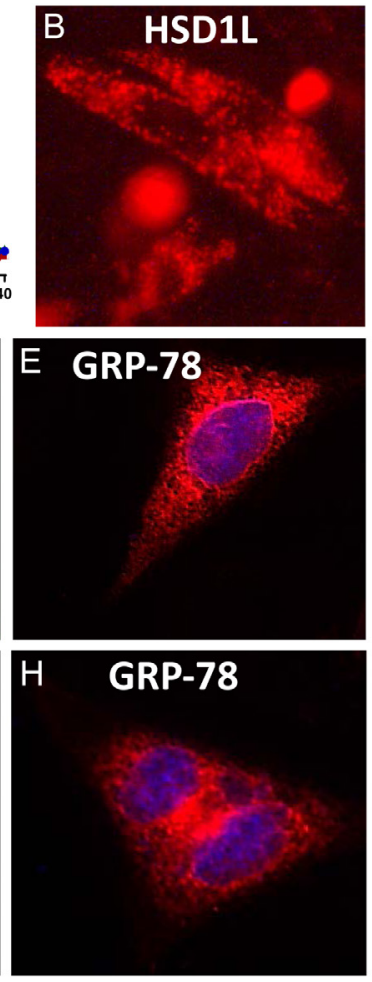
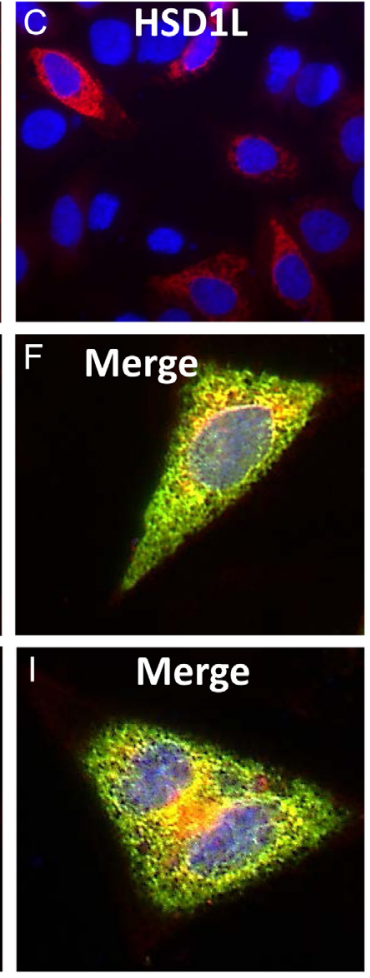

Figure 8

Intracellular localisation of HSD1L to the endoplasmic reticulum in transfected human HEK293 cells. (A) Predicted signal peptide score for the first 40 amino acids of human HSD1L (286 isoform) using SignallP4.1 software. C-Score (raw cleavage site score): S-Score (signal peptide score), Y-Score (combined cleavage site score). (B) Fluorescent image of C-terminal DS-redtagged human HSD11B1L9A transfected in HEK293 cells. (C) Full length human HSD11B1L transfected into HEK293 cells and then immunostained with an in-house mouse monoclonal anti-human HSD11B1L antibody and also anti-lgG alexa555 to stain nuclei. (D and G) Indirect immunofluorescence (green) for the known ER-localised protein GRP78. (E and H) Indirect immunofluorescence (red) for transfected human HSD1L in HEK293 cells using the mouse monoclonal anti-human HSD11B1L antibody. (F and I) Merged double-immunofluorescence (yellow) for transfected HSD1L (red) and endogenous GRP78 (green) in HEK293 cells. http://www.endocrineconnections.org DOI: 10.1530/EC-17-0119
(C) 2017 The authors Published by Bioscientifica Ltd

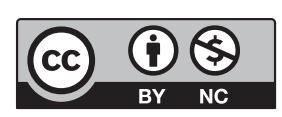

This work is licensed under a Creative Commons Attribution-NonCommercial 4.0 International License. 
SignalIP 4.1 revealed a predicted N-terminal hydrophobic ER localisation signal with a cleavage site predicted to reside between residues 15 and 16 of the protein (Fig. 8A). To further investigate the potential intracellular localisation of HSD1L to the ER, transfection experiments were performed in human HEK293 cells. Transfection of cells with HSD1L tagged with mCherry at the C-terminus showed a cytoplasmic localisation reminiscent of localisation to the ER (Fig. 8B). Indirect immunofluorescence of untagged HSD1L transfected into HEK293 cells using an anti-human HSD1L antibody showed a similar pattern of localisation (Fig. 8C). Finally, indirect immunofluorescence was performed for HSD1L and the ER marker GRP-78 in HEK393 cells transfected with HSD1L, and showed specific co-localisation of HSD1L to the cytoplasmic ER (Fig. 8, panels $\mathrm{F}$ and I, merged yellow fluorescence).

\section{Discussion}

The protein HSD1L has been identified as a member of the SDR superfamily of oxidoreductase enzymes with a high level of homology at the amino acid level to the very well characterised glucocorticoid steroid metabolising

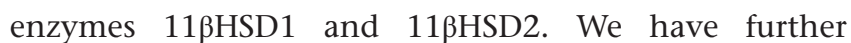
characterised HSD1L as a species-restricted member of the SDR superfamily strikingly absent from the majority of rodent genomes. We have compared the mRNA expression patterns of all three HSD-like SDR family members and identified cell type specific localization of HSD1L protein in a subset of specific tissues. Protein localization was cell type specific and was primarily localised to well characterised endocrine cells including gonadotrophs within the anterior pituitary and the steroid producing granulosa cells surrounding ovarian follicles. Further analysis in HSD1L transfected HEK293 cells showed that like the other family members, HSD1L is indeed an ER cytoplasmic localised protein. Recently published evidence have shown that HSD1L is unlikely to be involved in interconversion of cortisol and cortisone, nor metabolism of 11-keto or 11-hydroxy-testosterone (17), but given the high levels of sequence homology and tissue localisation, a steroid-based substrate is a likely target and will be the focus of future studies.

The presence of a third potential 11ßHSD enzyme in the sheep kidney was proposed in 1997 by GomezSanchez and coworkers (18) and was referred to as 11ß-HSD3 (18). Gomez-Sanchez and coworkers (18) isolated intact kidney microsomes and showed the presence of unidirectional conversion of cortisone/corticosterone that was NADP+ dependent with a $K_{\mathrm{m}}$ for corticosterone of $1.3 \mathrm{nM}$ (18), a 100-fold increase in activity compared to $11 \beta$ HSD1 in liver and other papers at the time that had described kinetics of 11 $\beta$ HSD1 from purified rat liver microsomes (19). However, this study was lacking in viable controls for the experiment with no confirmation that cortisol to cortisone reduction was not a result of $11 \beta \mathrm{HSD} 2$ activity given the well characterised high levels of $11 \beta$ HSD2 in the sheep kidney. This is especially true for $11 \beta$ HSD1 with specificity for cortisone both in vitro and in vivo relatively low, with some groups providing data to suggest that 11ßHSD1 has a higher affinity for cortisol over cortisone $(19,20,21)$. Other studies have shown that the specificity of $11 \beta \mathrm{HSD} 1$ towards cortisone is the result of co-expression of Hexose-6-Phosphate Dehydrogenase (H6PD) with 11ßHSD1 in the ER (22). The co-expression and localisation of HSD1L with H6PD has so far not been explored. A recent study has characterised a novel and potentially third $11 \beta \mathrm{HSD}$ activity in human liver nuclei that had lower $K_{\mathrm{m}}$ values for corticosterone, was NADP+ dependent and blocked by HSD1 inhibitors (23). This is however very unlikely to be HSD1L as we have shown that human HSD1L is not nuclear localised and has a very low level of expression in the sheep liver.

A more recent study by Ohno and coworkers (24) described HSD1L in the pig as a third HSD11B isozyme expressed in the neonatal pig testis (24). They showed by semi-quantitative PCR that a HSD1L mRNA was expressed in the neonatal pig testis and brain at lower levels compared to $11 \beta \mathrm{HSD} 1$ and $11 \beta \mathrm{HSD} 2$. The HSD1L activity in the testis was not inhibited by glycyrrhetinic acid, a strong inhibitor of $11 \beta \mathrm{HSD} 1$. These results are in agreement with our study that shows high expression of HSD1L in the brain and testis of non-human primates and sheep, with localised expression of marmoset HSD1L to

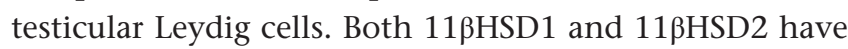
been characterised in the Leydig cells surrounding the seminiferous tubules in the testis of rats and opens up the question of the additional role of HSD1L that may play in the functioning of Leydig cell (25).

The results published by Huang and coworkers (10), further defined the expression and role of human HSD1L, termed SCDR10B, a member of the SDR enzyme family (10). They defined the intron/exon structure of the human HSD1L gene, showed strong expression in brain RNA by northern blot analysis and a very weak expression in other organs were analysed. Using expressed and purified HSD1L protein they demonstrated in vitro a very weak $11 \beta$-HSD dehydrogenase activity. Interestingly immunohistochemistry using an in-house polyclonal

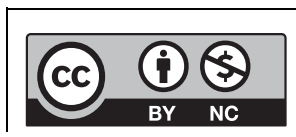

This work is licensed under a Creative Commons Attribution-NonCommercial 4.0 International License. 
antibody to human HSD1L indicated elevated expression in lung cancer biopsies and in various areas of the brain such as the hippocampus. We have found some evidence of elevated mRNA levels of human HSD1L in various cancer cell lines (unpublished results), but these results need to be explored in more detail to define a potential role for this enzyme in cancer cell proliferation. We have confirmed high expression of HSD1L in total RNA of the brain and show strong specific expression in the pituitary gland where HSD1L is localised specifically to gonadotrophs within the anterior pituitary. There are lower levels of expression in other regions of the nonhuman primate and sheep brain, yet the specific cell types expressing HSD1L are yet to be defined. In comparison to $11 \beta$ HSD 1 and 11 $\beta$ HSD2 in the foetal sheep, we also detect similar yet lower levels of HSD1L mRNA in the kidney, lung, adrenal and gastrointestinal tract.

Expression of HSD1L both at the mRNA and protein level in tissues of the hypothalamic-pituitary-gonadal (HPG) axis is striking and suggests a potential role for the enzyme in regulating aspects of reproduction. Expression of HSD1L in the anterior pituitary was shown to co-localise to gonadotroph cells through double labelling with LH, a well characterised cell marker of gonadotrophs. A major role of gonadotrophs in females is the cyclic release of $\mathrm{LH}$ and FSH, which in turn stimulates receptors on the surface of granulosa cells surrounding ovarian follicles. We intend to explore HSD1L-mediated regulation of LH and FSH production and release from pituitary gonadotrophs. This process is very important in the maintenance of reproductive homeostasis in both females and males and the expression of HSD1L in both ovarian granulosa cells and gonadotrophs provides evidence for a novel regulatory loop potentially regulated by HSD1L. This observation is also important with respect to its substrate given the importance role of granulosa cells for steroid production. Granulosa cells have been shown to upregulate important genes involved in steroid production including $\mathrm{p} 450$ aromatases, StAR and $17 \beta-\mathrm{HSD}$ in response to $\mathrm{LH}$ and FSH $(26,27)$. Increased levels of $\mathrm{LH}$ and FSH mediate stimulation of granulosa cells to increase serum levels of testosterone that are important substrates for aromatase enzymes present within theca cells responsible for the conversion of androgens to estradiol (28). Interestingly, the androgen receptor has been shown to be selectively expressed in rat granulosa cells acting as a mediator of positive feedback mechanisms (29). Furthermore, $11 \beta$ HSD 1 has been shown to bind and metabolise several androgenic derivatives $(30,31)$. This substrate promiscuity of $11 \beta \mathrm{HSD} 1$ may provide potential clues to the substrate of HSD1L given the high levels of sequence homology at a protein level. 11 $\beta$ HSDs are also able to metabolise 11-oxy androgens, such as 11-keto- and 11-hydroxy-testosterone $(32,33)$. A very recent study has investigated the steroids as substrates for HSD1L in zebrafish but showed absence of 11-ketosteroid reduction in zebrafish, with both human HSD1L (11 $\beta$ HSD3) and the two zebrafish homologues were unable to convert cortisone or 11-keto-testosterone to their 11 hydroxy metabolites (17). The identity of the physiological substrate for HSD1L/11 $\beta$ HSD3 is therefore elusive and may be an as yet uncharacterised reproductive steroid metabolite.

In summary, we have further characterised a novel species-restricted SDR hydroxysteroid dehydrogenase enzyme called HSD1L that is localised primarily to tissues of the pituitary-gonadal axis. The in vivo substrate for this enzyme is as yet unknown and given its level of expression in pituitary gonadatropes and the ovary, may serve as a modulator of reproductive function.

\section{Supplementary data}

This is linked to the online version of the paper at http://dx.doi.org/10.1530/ EC-17-0119.

\section{Declaration of interest}

The authors declare that there is no conflict of interest that could be perceived as prejudicing the impartiality of the research reported.

\section{Funding}

This research did not receive any specific grant from any outside funding agency in the public, commercial or not-for-profit sector. Spencer Greatorex was funded by a Monash University MBio PhD scholarship. This work was partly funded by the Monash University Outside-Study Programme.

\section{Acknowledgements}

The authors would like to thank Judy $\mathrm{Ng}$ and $\mathrm{Dr}$ Robert De Matteo for technical assistance.

\section{References}

1 Kallberg Y, Oppermann U, Jornvall H \& Persson B. Short-chain dehydrogenase/reductase (SDR) relationships: a large family with eight clusters common to human, animal, and plant genomes. Protein Sciences 200211 636-641. (doi:10.1110/ps.26902)

2 Persson B, Kallberg Y, Bray JE, Bruford E, Dellaporta SL, Favia AD, Duarte RG, Jornvall H, Kavanagh KL, Kedishvili N, et al. The SDR (short-chain dehydrogenase/reductase and related enzymes) nomenclature initiative. Chemico-Biological Interactions 2009178 94-98. (doi:10.1016/j.cbi.2008.10.040)

3 Jornvall H, Hedlund J, Bergman T, Kallberg Y, Cederlund E \& Persson B. Origin and evolution of medium chain alcohol dehydrogenases. ChemicoBiological Interactions 2013202 91-96. (doi:10.1016/j.cbi.2012.11.008)

4 Kallberg Y, Oppermann U, Jornvall H \& Persson B. Short-chain dehydrogenases/reductases (SDRs). European Journal of Biochemistry 2002269 4409-4417. (doi:10.1046/j.1432-1033.2002.03130.x)

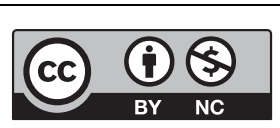

This work is licensed under a Creative Commons Attribution-NonCommercial 4.0 International License. 
5 Chapman K, Holmes M \& Seckl J. 11beta-Hydroxysteroid dehydrogenases: intracellular gate-keepers of tissue glucocorticoid action. Physiological Reviews 201393 1139-1206. (doi:10.1152/ physrev.00020.2012)

6 Agarwal AK, Rogerson FM, Mune T \& White PC. Gene structure and chromosomal localization of the human HSD11K gene encoding the kidney (type 2) isozyme of 11 beta-hydroxysteroid dehydrogenase. Genomics 199529 195-199. (doi:10.1006/geno.1995.1231)

7 Krozowski Z, Li KX, Koyama K, Smith RE, Obeyesekere VR, SteinOakley A, Sasano H, Coulter C, Cole T \& Sheppard KE. The type I and type II 11beta-hydroxysteroid dehydrogenase enzymes. Journal of Steroid Biochemistry and Molecular Biology 199969 391-401. (doi:10.1016/S0960-0760(99)00074-6)

8 Morgan SA, McCabe EL, Gathercole L, Hassan-Smith ZK, Larner DP, Bujalska IJ, Stewart PM, Tomlinson JW \& Lavery GG. 11 $\beta$-HSD1 is the major regulator of the tissue-specific effects of circulating glucocorticoid excess. PNAS 2014111 E2482-E2491. (doi:10.1073/pnas.1323681111)

9 Gathercole LL, Lavery GG, Morgan SA, Cooper MS, Sinclair AJ, Tomlinson JW \& Stewart PM. 11beta-Hydroxysteroid dehydrogenase 1: translational and therapeutic aspects. Endocrine Reviews 201334 525-555. (doi:10.1073/pnas.1323681111)

10 Huang C, Wan B, Gao B, Hexige S \& Yu L. Isolation and characterization of novel human short-chain dehydrogenase/ reductase SCDR10B which is highly expressed in the brain and acts as hydroxysteroid dehydrogenase. Acta Biochimica Polonica 200956 279-289. (doi:10.1016/j.bbagrm.2008.09.005)

11 Baker ME. Evolution of 11beta-hydroxysteroid dehydrogenase-type 1 and 11beta-hydroxysteroid dehydrogenase-type 3. FEBS Letters 2010 584 2279-2284. (doi:10.1016/j.febslet.2010.03.036)

12 Sousa JF, Torrieri R, Silva RR, Pereira CG, Valente V, Torrieri E, Peronni KC, Martins W, Muto N, Francisco G, et al. Novel primatespecific genes, RMEL 1, 2 and 3, with highly restricted expression in melanoma, assessed by new data mining tool. PLOS ONE 20105 e13510. (doi:10.1371/journal.pone.0013510)

13 Takamatsu K, Maekawa K, Togashi T, Choi DK, Suzuki Y, Taylor TD, Toyoda A, Sugano S, Fujiyama A, Hattori M, et al. 2002 Identification of two novel primate-specific genes in DSCR. DNA Research 20099 89-97. (doi:10.1093/dnares/9.3.89)

14 Tay SK, Blythe J \& Lipovich L. Global discovery of primate-specific genes in the human genome. PNAS 2009106 12019-12024. (doi:10.1073/pnas.0904569106)

15 Pfaffl MW. A new mathematical model for relative quantification in real-time RT-PCR. Nucleic Acids Research 200129 e45. (doi:10.1093/ nar/29.9.e45)

16 Zhang J, Osslund TD, Plant MH, Clogston CL, Nybo RE, Xiong F, Delaney JM \& Jordan SR. Crystal structure of murine 11 betahydroxysteroid dehydrogenase 1: an important therapeutic target for diabetes. Biochemistry 200544 6948-6957. (doi:10.1021/bi047599q)

17 Tsachaki M, Meyer A, Weger B, Kratschmar DV, Tokarz J, Adamski J, Belting HG, Affolter M, Dickmeis T \& Odermatt A. Absence of 11-keto reduction of cortisone and 11-ketotestosterone in the model organism zebrafish. Journal of Endocrinology 2017232 323-335. (doi:10.1530/ JOE-16-0495)

18 Gomez-Sanchez EP, Ganjam V, Chen YJ, Cox DL, Zhou MY, Thanigaraj S \& Gomez-Sanchez CE. The sheep kidney contains a novel unidirectional, high affinity NADP(+)-dependent 11 betahydroxysteroid dehydrogenase (11 beta-HSD-3). Steroids 199762 444-450. (doi:10.1016/S0039-128X(97)00011-1)

19 Lakshmi V \& Monder C. Purification and characterization of the corticosteroid 11 beta-dehydrogenase component of the rat liver 11 beta-hydroxysteroid dehydrogenase complex. Endocrinology 1988123 2390-2398. (doi:10.1210/endo-123-5-2390)
20 Condon J, Ricketts ML, Whorwood CB \& Stewart PM. Ontogeny and sexual dimorphic expression of mouse type 2 11beta-hydroxysteroid dehydrogenase. Molecular and Cellular Endocrinology 1997127 121-128. (doi:10.1016/S0303-7207(97)04000-8)

21 Hult M, Jornvall H \& Oppermann UC. Selective inhibition of human type 1 11beta-hydroxysteroid dehydrogenase by synthetic steroids and xenobiotics. FEBS Letters 1998441 25-28. (doi:10.1016/S00145793(98)01515-4)

22 Bujalska IJ, Draper N, Michailidou Z, Tomlinson JW, White PC, Chapman KE, Walker EA \& Stewart PM. Hexose-6-phosphate dehydrogenase confers oxo-reductase activity upon 11 betahydroxysteroid dehydrogenase type 1. Journal of Molecular Endocrinology 200534 675-684. (doi:10.1677/jme.1.01718)

23 Robinzon B \& Prough RA. A novel NADP(+)-dependent dehydrogenase activity for 7alpha/beta- and 11beta-hydroxysteroids in human liver nuclei: a third 11beta-hydroxysteroid dehydrogenase. Archives of Biochemistry and Biophysics 2009486 170-176. (doi:10.1016/j.abb.2009.04.010)

24 Ohno S, Nakagawara S, Honda Y \& Nakajin S. Evidence for expression of 11beta-hydroxysteroid dehydrogenase type3 (HSD11B3/HSD11B1L) in neonatal pig testis. Molecular and Cellular Biochemistry 2013381 145-156. (doi:10.1007/s11010-013-1697-4)

25 Zhou HY, Chen XX, Lin H, Fei AL \& Ge RS. 11beta-Hydroxysteroid dehydrogenase types 1 and 2 in postnatal development of rat testis: gene expression, localization and regulation by luteinizing hormone and androgens. Asian Journal of Andrology 201416 811-816. (doi:10.4103/1008-682X.132471)

26 Minegishi T, Tsuchiya M, Hirakawa T, Abe K, Inoue K, Mizutani T \& Miyamoto K. Expression of steroidogenic acute regulatory protein (StAR) in rat granulosa cells. Life Science 200067 1015-1024. (doi:10.1016/S0024-3205(00)00696-2)

27 Richards JS, Jonassen JA, Rolfes AI, Kersey K \& Reichert LE Jr. Adenosine 3',5'-monophosphate, luteinizing hormone receptor, and progesterone during granulosa cell differentiation: effects of estradiol and follicle-stimulating hormone. Endocrinology 1979104 765-773. (doi:10.1210/endo-104-3-765)

28 Erickson GF \& Hsueh AJ. Stimulation of aromatase activity by follicle stimulating hormone in rat granulosa cells in vivo and in vitro. Endocrinology 1978102 1275-1282. (doi:10.1210/endo-102-4-1275)

29 Tetsuka M \& Hillier SG. Androgen receptor gene expression in rat granulosa cells: the role of follicle-stimulating hormone and steroid hormones. Endocrinology 1996137 4392-4397. (doi:10.1210/ endo.137.10.8828500)

30 Odermatt A \& Klusonova P. 11beta-Hydroxysteroid dehydrogenase 1: regeneration of active glucocorticoids is only part of the story. Journal of Steroid Biochemistry and Molecular Biology 2015151 85-92. (doi:10.1016/j.jsbmb.2014.08.011)

31 Odermatt A \& Kratschmar DV. Tissue-specific modulation of mineralocorticoid receptor function by 11beta-hydroxysteroid dehydrogenases: an overview. Molecular and Cellular Endocrinology 2012350 168-186. (doi:10.1016/j.mce.2011.07.020)

32 Storbeck KH, Bloem LM, Africander D, Schloms L, Swart P \& Swart AC. 11beta-Hydroxydihydrotestosterone and 11-ketodihydrotestosterone, novel C19 steroids with androgenic activity: a putative role in castration resistant prostate cancer? Molecular and Cellular Endocrinology 2013377 135-146. (doi:10.1016/j.mce.2013.07.006)

33 Swart AC, Schloms L, Storbeck KH, Bloem LM, Toit T, Quanson JL, Rainey WE \& Swart P. 11beta-Hydroxyandrostenedione, the product of androstenedione metabolism in the adrenal, is metabolized in LNCaP cells by 5alpha-reductase yielding 11beta-hydroxy-5alphaandrostanedione. Journal of Steroid Biochemistry and Molecular Biology 2013138 132-142. (doi:10.1016/j.jsbmb.2013.04.010)

Received in final form 1 August 2017

Accepted 7 August 2017
This work is licensed under a Creative Commons Attribution-NonCommercial 4.0 International License. 